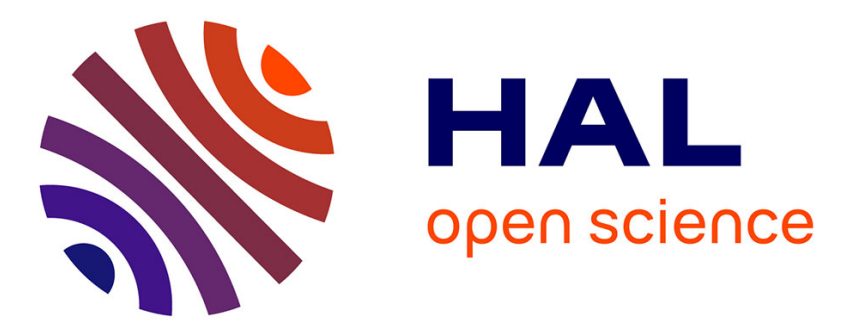

\title{
Microfluidic Assisted Flash Precipitation of Photocrosslinkable Fluorescent Organic Nanoparticles for Fine Size Tuning and Enhanced Photoinduced Processes
}

Stéphane Hoang, Simon Olivier, Stéphane Cuenot, Agnès Montillet, Jérôme Bellettre, Eléna Ishow

\section{To cite this version:}

Stéphane Hoang, Simon Olivier, Stéphane Cuenot, Agnès Montillet, Jérôme Bellettre, et al.. Microfluidic Assisted Flash Precipitation of Photocrosslinkable Fluorescent Organic Nanoparticles for Fine Size Tuning and Enhanced Photoinduced Processes. ChemPhysChem, 2020, 10.1002/cphc.202000633 . hal-03011586

\author{
HAL Id: hal-03011586 \\ https://hal.science/hal-03011586
}

Submitted on 26 Feb 2021

HAL is a multi-disciplinary open access archive for the deposit and dissemination of scientific research documents, whether they are published or not. The documents may come from teaching and research institutions in France or abroad, or from public or private research centers.
L'archive ouverte pluridisciplinaire $\mathbf{H A L}$, est destinée au dépôt et à la diffusion de documents scientifiques de niveau recherche, publiés ou non, émanant des établissements d'enseignement et de recherche français ou étrangers, des laboratoires publics ou privés. 


\title{
Microfluidic Assisted Flash Precipitation of Photocrosslinkable
}

\section{Fluorescent Organic Nanoparticles for Fine Size Tuning and}

\section{Enhanced Photoinduced Processes}

\author{
Stéphane Hoang, ${ }^{[\mathrm{a}]}$ Simon Olivier, ${ }^{[\mathrm{a}],[\mathrm{b}]}$ Stéphane Cuenot, ${ }^{[\mathrm{c}]}$ Agnès Montillet, ${ }^{[\mathrm{d}]}$ Jérôme \\ Bellettre, ${ }^{[\mathrm{e}]}$ and Eléna Ishow ${ }^{*[\mathrm{a}]}$
}

\author{
[a] S. Hoang, Dr. S. Olivier, Pr. E. Ishow \\ CEISAM-UMR CNRS 6230, Université de Nantes, 2 rue de la Houssinière, 44322 Nantes, France \\ E-mail: elena.ishow@univ-nantes.fr \\ [b] New affiliation: Dr. S. Olivier, Air Liquide, Air Liquide Facility, 28 Wadai, Tsukuba, Ibaraki 300-4247, Japan \\ [c] Dr. S. Cuenot \\ Université de Nantes, CNRS, Institut des Matériaux Jean Rouxel, IMN, F-44000 Nantes, France \\ [d] Pr. A. Montillet \\ GEPEA UMR CNRS 6144, IUT Saint Nazaire, Université de Nantes, 58 rue Michel Ange, 44600 Saint Nazaire, \\ France \\ [e] Pr. J. Bellettre \\ LTeN UMR CNRS 6607, Polytech Nantes, Université de Nantes, rue Christian Pauc, 44306 Nantes, France
}

\begin{abstract}
Highly concentrated dispersions of fluorescent organic nanoparticles (FONs), broadly used for optical tracking, bioimaging and drug delivery monitoring, are obtained using a newly designed micromixer chamber involving high impacting flows. Fine size tuning and narrow size distributions are easily obtained by varying independently the flow rates of the injected fluids and the concentration of the dye stock solution. The flash nanoprecipitation process employed herein is successfully applied to the fabrication of bicomposite FONs designed to allow energy transfer. Considerable enhancement of the emission signal of the energy acceptors is promoted and its origin revisited as a result of polarity rather than steric effects. Finally, we exploit the high spatial confinement encountered in FONs and their ability to
\end{abstract}


encapsulate hydrophobic photosensitizers to induce photocrosslinking. Increase in the photocrosslinked FON stiffness is evidenced $\mathrm{d}$ by measuring the elastic modulus at the nanoscale using atomic force microscopy. Such results pave the way toward the straightforward fabrication of multifunctional and mechanically photoswitchable FONs, opening novel potentialities in terms of sensing, multimodal imaging, and theranostics.

\section{INTRODUCTION}

For the past two decades, the technical development of scientific instruments has been accompanied by an increasing demand of miniaturization to gain spatial and time resolution and access to novel phenomena related to size reduction down to the nanometer scale. Assessing flow velocimetry in microfluidics, ${ }^{[1]}$ sensing local temperatures ${ }^{[2]}, \mathrm{pH}^{[3]}$ or chemicals ${ }^{[4]}$, and tracking cell $^{[5]}$ or nanomedicine ${ }^{[6]}$ require nanometer-sized materials to follow local evolutions as a function of external parameters. In this context, emissive nanoparticles have been considered as attractive tools since they can easily been monitored by widespread wide-field or confocal fluorescence microscopes, offering high detection sensitivity and versatile implementation of the investigated devices or (bio)chemical systems. ${ }^{[7]}$ The search for bright but biodegradable and heavy-metal free nanoparticles is thus becoming a pressing demand, requiring progressive replacement of the broadly used luminescent silica nanoparticles and quantum dots, facing the abovementioned limitations. For these reasons, engineering bright fluorescent organic nanoparticles with large colloidal stability, low polydispersity index and additional functionality, stimulates a large area of investigations. Fluorescent nanolatexes, namely submicrometer-sized crosslinked polymer particles incorporating fluorophores, represent the most regarded family of 
organic nanoparticles. Usually, they are synthesized by resorting to emulsion or dispersion-based fabrication processes. ${ }^{[8]}$ Their transformation into fluorescent nanolabels can be achieved by entrapping fluorophores before polymerization, ${ }^{[9]}$ or by inducing self-assembling of preformed amphiphilic block copolymers in the presence of hydrophilic or hydrophobic fluorophores. ${ }^{[10]}$ Another approach relies on polymer networks incorporating fluorophores as covalent side chains, which requires complementary reactive functions. ${ }^{[11]}$ However, the first approach faces extensive dye release in the aqueous media in the long term, especially for ionic and hydrosoluble fluorophores, thereby leading to false-positive. As for the second approach, the risk of selfquenching upon aggregation for hydrophobic dyes or the natural electrostatic repulsions between dyes of similar charges requires diluted dyes concentrations, leading to low-payload nanolabels. In this case, prolonged irradiation tends to conduct to the rapid loss of emission signal due to the weak density of dyes, amenable to photobleaching in the presence of singlet oxygen. To circumvent this issue of a low functionalization rate, an alternative approach has been proposed, based on the formation of non-doped fluorescent nanoparticles, resulting from the self-assembly of a high amount of small hydrophobic and fluorescent molecules, promoted by water addition. Two main structural strategies have been developed to still obtain emission after dye aggregation into nanoparticles. The first one led to the coined aggregation-induced-emissive (AIE) nanomaterials where emission arises from blocked intramolecular rotations of the dyes within the nanoparticles upon $\pi-\pi$ stacking, ${ }^{[12]}$ or from the formation of $\mathrm{H}$ - and $\mathrm{J}$-aggregates. ${ }^{[13]}$ The second one involves bulky or twisted fluorophores that emit in solution and keep emitting as nanoparticles thanks to the large intermolecular distance introduced by sterically hindered substituents, prohibiting the formation of dark $\pi-\pi$ states. ${ }^{[14]}$ Whatever the adopted synthetic method, the resulting fluorescent organic nanoparticles (FONs) are formed upon solvent shifting, 
namely essentially through manual injection into a large volume of water of a diluted solution of fluorophores dissolved in a water-miscible solvent. Such a fabrication process tightly depends on the operator and geometry of the precipitation container, which suffers from diluted solutions, small preparation volumes, and a lack of reproducibility and size control. To solve such issues, smart micromixers have been developed to ensure quick mixing and achieve high supersaturation and nucleation rate that are prerequisites for nanoparticle formation in the millisecond range following a nucleation-growth or nucleation-aggregation process. ${ }^{[15]}$ Two mixer geometries have been proposed by B. K Johnson and R. K. Prud'homme et al., named confined impinging jet (CIJ) mixer ${ }^{[16]}$ and multi-inlet vortex mixer (MIVM) ${ }^{[17]}$ to produce high turbulence, favoured by a high flow velocity or large Reynolds numbers above 1000. CIJ mixers consist in two sub-millimeter channels, placed face-to-face across a large mixing chamber and connected to gas-tight syringes that contain each water or organic solution and are attached with each other to exert equal injecting pressure (Figure S1A). Although CIJ mixers are simple and hand-operated, the momenta of the organic solution and anti-solvent (here water) are necessarily identical, limiting their contribution to the control of the resulting nanoparticle diameters and the use of concentrated solutions at the risk of causing dye precipitation. MVIM systems overcome these restrictions thanks to a conical mixing chamber connected to two or four flow inlets, placed in a symmetrical and tangent manner around the chamber, which affords separate control of the stream velocity, continuous fluorescent nanoparticle production and easy variation of the solution concentration (Figure S1B-C). ${ }^{[18]}$ Although both CIJ and MVIM technical solutions are elegant, the mixer dimensions are still too large to be implemented in the future on a microscope stage and allow for straightforward optical follow-up to gain understanding in the mixing dynamics. Moreover, they all imply the use of surfactants or amphiphilic polymers in large concentrations 
to stabilize the generated nanoparticles, ${ }^{[10 \mathrm{~b}]}$ which can impact the flow dynamics and provide dyefree micelles in the case of bad mixing. In this respect, a novel compact micromixer, designated as Microsphere500, has been developed in the past for the fabrication of surfactant-free oil-in water emulsions at high flow rates and under highly impacting flows. ${ }^{[19]}$ Its original geometry, largely detailed in previous studies, ${ }^{[19]}$ is based on two opposing channels with distinct sections, enabling high-impact impinging flows. The mutual position of the channels, in a slight offset with regard to the longitudinal stream direction, creates a vortex stream that superimposes the flow instabilities due to the high energy impact, hence very efficient mixing of biphasic mixtures is obtained. ${ }^{[20]}$ We thus want to demonstrate that FONs with controllable diameter can easily be obtained out of small fluorophores by resorting to the simple Microsphere500 micromixer, combining the advantages of both CIJ and MVIM systems, namely high flow momenta, continuous nanoparticle production and technical simplicity. Issues regarding FON homogeneity and composition as a function of the experimental conditions of fabrication are addressed by steady-state and time-resolved photophysical studies. To validate the versatility of the current microfluidic device, we show that bicomposite FONs can be obtained by flash coprecipitation, allowing for tunable emission color thanks to the large structural confinement effects encountered in FONs and responsible for efficient photoinduced electronic energy or electron transfer. ${ }^{[21]}$ Finally, we harness such confinement effects to show the possibility of chemical reaction within pre-formed FONs. To this aim, we perform photocrosslinking assays using FONs comprising bisacrylate fluorescent monomers and tetrathiol crosslinker to offer in the future easily accessible emissive nanotracers as an alternative to crosslinked polystyrene and silica nanoparticles. ${ }^{[22]}$ 


\section{EXPERIMENTAL SECTION}

\subsection{Chemicals and solution preparation}

Both fluorophores employed in these studies, ALD (bis(2-(acryloyloxy)ethyl) O,O'-(((4formylphenyl)azanediyl)bis([1,1'-biphenyl]-4',4-diyl))bis(ethane-1,1-diyl)) disuccinate) and VIN (bis(2-(acryloyloxy)ethyl) O,O'-(((4-(2,2-dicyanovinyl)phenyl)azanediyl)bis([1,1'biphenyl]-4',4-diyl))bis(ethane-1,1-diyl)) disuccinate), were synthesized according to literature procedure. ${ }^{[23]}$ Spectroscopic grade and inhibitor-free THF, used for all preparations and photophysical studies, and photocrosslinking respectively were purchased from Aldrich. Three ALD solutions in THF with varying concentrations $(0.5,1$ and 2 wt. \%) were initially prepared to cover all flash nanoprecipitation conditions. The bicomposite solutions comprising ALD and VIN to generate FONs with tunable emission wavelength were prepared from two separate stock solutions in THF, containing each ALD and VIN at a 0.5 wt. \% concentration. The final solutions $(2 \mathrm{~mL})$ to be injected in the micromixer were prepared by adjusting the required volumes of the ALD and VIN stock solutions and completing with $1 \mathrm{~mL}$ of THF, so that distinct ALD:VIN ratios of 100:0, 95:5, 80:20 and 0:100 could be obtained with a total dye concentration fixed at 0.25 wt. $\%$.

\subsection{Flash nanoprecipitation}

Manual method involving magnetic stirring. A solution of organic compounds $(100 \mu \mathrm{L})$ was added in one shot with a micropipette (Acura ${ }^{\circledR}$ manual 825$)$ into a $18 \mathrm{~mL}$-large amber glass vial, containing water $(2.5 \mathrm{~mL})$ while stirring with a vortex mixer (IKA VORTEX Genius 3 ) to afford FONs. 
Method involving the Microsphere500 micromixer. The nanoparticles were fabricated via a microfluidic circuit including the Microsphere $500^{[19]}$ mixing chamber made from aluminum plates, pump syringes from Harvard Apparatus (Pump Elite 11) and single-use plastic syringes 2 or $5 \mathrm{~mL}$, and $50 \mathrm{~mL}$ (HSW SOFT-Ject ${ }^{\circledR}$ Luer Lock) connected to the mixing chamber via Teflon tubing. The two smallest syringes were used for the fluorophore solution in THF and the $50 \mathrm{~mL}$ one for water. The organic solution was injected through the smaller square channel $(300 \mu \mathrm{m}$ wide) of the microcircuit while water was injected through the larger square channel $(600 \mu \mathrm{m}$ wide). Solution volumes of $2 \mathrm{~mL}$ were at least needed for each measurement due to the dead volume. FON dispersions were collected from the mixing chamber outlet via two Teflon tubes, placed in mutual opposite directions The microfluidic circuit was cleaned multiple times with THF and water between each measurement, except for experiment A where the concentrations were the same for every sample. The Microsphere500 mixing chamber could be used as many times as needed, and tubes were renewed only if fluorophore traces remained after thorough washing.

\subsection{Photocrosslinking reaction}

Preparation of solutions for photopolymerization as FONs and thin films. Three distinct solutions of ALD (15.0 mg, $3.3 \mathrm{mmol}$ ), pentaerythritoltetrakis(3-mercaptoprioionate) (PT-SH) (8.1 mg, $3.3 \mathrm{mmol}$ ), and 2,4-6-trimethylbenzoyl-diphenylphosphine (TPO) (1.4 mg, $0.8 \mathrm{mmol}$ ) in THF (5 $\mathrm{mL}$ ) were separately prepared. Three daughter solutions (A and B), completed to a $2 \mathrm{~mL}$ total volume with THF were made out of them following the TPO:PT-SH:ALD volume composition (in $\mathrm{mL}$ ) as follows: P0) 0:0:1 in THF (1 mL), P1) 0.1:0.1:1 in THF (0.8 mL) and P5) 0.1:0.5:1 in THF $(0.4 \mathrm{~mL})$. Such solutions were directly deposited on a glass slide and let dry to form homogeneous thin films. Alternatively, $\mathrm{FON}_{0}, \mathrm{FON}_{0.1}$ and $\mathrm{FON}_{0.5}$ could be produced by manual 
addition in water $(2.5 \mathrm{~mL})$ under vortex stirring of a small amount $(100 \mu \mathrm{L})$ of solutions $\mathrm{P} 0, \mathrm{P} 1$ or $\mathrm{P} 5$ respectively.

Photocrosslinking conditions. Photocrosslinking was performed in $1 \mathrm{~cm}$-wide quartz cell under constant stirring, using a UV light source Xe-Hg lamp (Hamamatsu LC8) paired with a $365 \mathrm{~nm}$ bandpass filter. The lamp power used for all experiments was set at $30 \mathrm{~mW} . \mathrm{cm}^{-2}$ and measured with a photodetector (UV-vis Nova II Ophir). Irradiation was performed for 5 min. Photocrosslinking in deoxygenated conditions was carried out via argon bubbling for 1 min. Then, a constant flow of argon was settled in the quartz cell for 2 min before photoirradiating the solution.

Photoirradiation of FONs, prepared here upon manual precipitation of a TPO:PT-SH:ALD mixture into water, was performed at $365 \mathrm{~nm}$ in a quartz cell under constant stirring using an irradiation lamp equipped with an interferential filter. FONs devoid of TPO or PT-SH were also prepared to serve as control samples. Two aeration conditions were examined: without and with preliminary deoxygenation for 2 min by argon bubbling of the FON suspensions. We fixed the irradiation time and power at 5 minutes and $30 \mathrm{~mW} \cdot \mathrm{cm}^{-2}$ respectively, which offered the best compromise in terms of photocrosslinking efficiency and persistence of fluorescence from preliminary solid-state experiments. After irradiation, all samples were diluted in the same way to yield absorbance such that the risks of inner filter effects in emission (less than 0.1 where the emission and absorption spectra overlap) and photoinduced photopolymerization during excitation (excitation slits with limited width were set) were minimized. 


\subsection{FON structural investigations}

Dynamic light scattering (DLS). FON size measurements in aqueous dispersions were performed via DLS using the VASCO Kin ${ }^{\mathrm{TM}}$ nanoparticle sizer provided by Cordouan Technologies, and equipped with equipped with a $45 \mathrm{~mW}$ diode laser operating at $638 \mathrm{~nm}$. The laser power was manually adjusted for each sample before any measurement to get satisfying results. All measurements were collected on aqueous FON dispersions at $25^{\circ} \mathrm{C}$ in a backscattering mode at a $170^{\circ}$ angle, over $2 \mathrm{~min}$ with a $5 \mathrm{~Hz}$ acquisition frequency, namely every $200 \mathrm{~ms}$. Acquisitions and analyses were done with the Nano $\mathrm{Kin}^{\mathrm{TM}}$ software, provided by Cordouan Technologies, Cumulant-based algorithm in intensity was privileged to infer size distributions. A logarithmic scale was adopted for clarity reason, improving visualization of all samples on the same scale, with no impact on the fitted parameters.

Zetametry. Measurements of surface potential $\zeta$, were carried out by means of a Zetasizer Nano ZS ZEN 3600 (Malvern). The samples were placed in disposable folded capillary cells (DTS1070). Two sets of three measurements were realized for each sample and the zeta potential was calculated from electrophoretic mobility dispersion fitted by the Smoluchowski model.

Transmission electron microscopy. FON size measurements as dry samples were performed using a MO-Jeol 1230 microscope $(80 \mathrm{kV})$ and diluted samples deposited on holey carbon-coated copper grids (300 mesh), purchased from Agar Scientific.

\subsection{Photophysical studies}

Steady-state measurements. UV-visible absorption spectra were recorded using a Varian Cary 5E spectrophotometer (Agilent). Emission spectra were recorded using a Fluorolog 3 spectrofluorometer (Horiba). Correction for the emission spectra with regard to the spectral 
response of the detector was automatically applied. Fluorescence quantum yields were determined in solution, referred to coumarine $540 \mathrm{~A}$ in ethanol $\left(\Phi_{f}=0.38\right)$.

Time-resolved fluorescence measurements. Fluorescence time decays were measured in water using the fully automated spectrofluorometer (model Fluotime 300, PicoQuant) following the time-correlated single photon counting method. Excitation was performed using a pulsed laser diode (LDH-D-C-450B) working at $450 \pm 10 \mathrm{~nm}$ (70 ps fwhm time resolution), or a pulsed LED (PLS 340) working at $340 \mathrm{~nm} \pm 10 \mathrm{~nm}$ (500 ps fwhm time resolution). Fluorescence decays were recorded using a Hybrid-PMT detector combined with an acquisition temporal resolution up to 25 ps. Long-pass edged filter purchased from Semrock centered at $473 \mathrm{~nm}$ (BPL01-473R-25) or $355 \mathrm{~nm}$ (BPL01-355R-25) was used to discard any possible contribution of excitation light scattering by the organic nanoparticle dispersions. Time fluorescence decay modeling was performed with EasyTau 1 software developed by PicoQuant.

Resonance energy transfer (RET). Förster resonance energy transfer could reasonably be evoked to explain the spectral evolution of the emission signals. The Förster radius can be estimated from the spectral overlap factor $J$ between the energy donor (ALD) and acceptor (VIN) (Eq. 2).

$\mathrm{R}_{0}^{6}=\frac{0.2108 \kappa^{2} \Phi^{\circ}{ }_{\mathrm{D}}^{\infty}}{\mathrm{n}^{4}} \int_{0}^{\infty} \mathrm{I}_{\mathrm{D}}(\lambda) \varepsilon_{\mathrm{A}}(\lambda) \lambda^{4} \mathrm{~d} \lambda \quad$ with $J=\int_{0}^{\infty} \mathrm{I}_{\mathrm{D}}(\lambda) \varepsilon_{\mathrm{A}}(\lambda) \lambda^{4} \mathrm{~d} \lambda$

$\Phi^{\circ}$ : fluorescence quantum yield of the donor in the absence of acceptor, n: refractive index of the solution, $\kappa^{2}$ : orientation factor with $\kappa^{2}=0.476$ for dipoles in a rigid medium like FONs, ID: emission intensity of the donor, $\varepsilon$ A: molar absorption coefficient of the acceptor. 
The energy transfer quantum efficiency $\Phi_{\text {FRET }}$ was calculated from the donor emission signal, through the expression $\Phi_{\mathrm{FRET}}=1-\mathrm{I}_{\mathrm{D}} / \mathrm{I}^{\circ} \mathrm{D}$, where $I^{\circ} \mathrm{D}$ and $I_{\mathrm{D}}$ feature the emission intensity in the absence and presence of acceptor, respectively.

\subsection{AFM quantitative mechanical measurements}

Sample preparation. Freshly cleaved mica squared plates (1 inch, Bruker) were treated with a $0.01 \%(\mathrm{w} / \mathrm{v})$ poly-L-lysine solution (Aldrich) for $15 \mathrm{~min}$, thoroughly rinsed with Millipore water and finally dried under a nitrogen flow. They were subsequently incubated for 30 min with FON dispersions that were previously diluted 100 times, and washed again with Millipore water. The dispersions were let dry in air before AFM measurements.

Atomic force microscopy (AFM). All AFM experiments were performed at room temperature (20 ${ }^{\circ} \mathrm{C}$ ) using a NanoWizard $\AA$ atomic force microscope (JPK, Germany) equipped with inverted optical microscope and $\mathrm{x}-\mathrm{y}$ closed-loop control system. Cantilevers with calibrated spherical carbon-glassy tips (Biosphere ${ }^{\mathrm{TM}}$ series purchased from Nanotools) characterized by a $100 \mathrm{~nm} \pm$ $10 \mathrm{~nm}$ tip radius and $2.8 \mathrm{~N} \cdot \mathrm{m}^{-1}$ spring constant were first used for imaging in intermittent contact mode to select nanoparticles to be probed. Force mapping using a scanning mode was privileged over repeated measurements on single nanoparticles to avoid tip positioning uncertainty relative to the nanoparticles. It offers the additional advantage to specifically select the force curves recorded at the nanoparticle center in a post-treatment procedure. Typically, $32 \times 32$ force mapping curves were recorded around the selected nanoparticle $(500 \mathrm{~nm} \times 500 \mathrm{~nm})$ and force versus piezo movement (F-Z) curves, located at the nanoparticle center, were extracted in an offline procedure. The raw F-Z approach curves were converted into force versus tip-sample distance curves to apply a contact mechanics model for a sphere-sphere contact geometry. The 
resulting apparent elastic modulus $E$ was then obtained by fitting these curves with the Hertz model.

\section{RESULTS AND DISCUSSION}

\subsection{FON fabrication: manual addition $v$ s microfluidic mixing chamber}

In order to generate photocrosslinkable FONs out of small molecules following high impact-flow microfluidics, we resorted to push-pull structures ALD and VIN that had been designed to display both fluorescence and photocrosslinking properties in the solid state for solution-processed OLEDs (Figure 1). ${ }^{[23-24]}$ Such compounds display large structural similarity and distinct emission ranges, namely greenish-blue for ALD and orange-red for VIN as a result of their aldehyde and dicyanovinylidenyl groups respectively, that will be exploited latter in the manuscript to fabricate color-tunable FONs.<smiles>[R]OC(C)c1ccc(-c2ccc(N(c3ccc(-c4ccc(C(C)O)cc4)cc3)c3ccc(C(C)O)cc3)cc2)cc1</smiles><smiles>O=C(CS)OCC(COC(=O)CS)(COC(=O)CS)COC(=O)CS</smiles><smiles>[R]CC(=O)CCC(=O)OCCOC(=O)C=C</smiles><smiles>Cc1cc(C)c(P(=O)(Oc2ccccc2)c2ccccc2)c(C)c1</smiles>

Figure 1. Structures of fluorophores processed as color-tunable and photocrosslinkable FONs. 
The propeller shape of the triphenylamino core and the introduction of two unresolved chiral centers at the benzylic carbon atoms, yield racemic mixtures, and advantageously limit the formation of dark $\pi-\pi$ aggregates. In this way, fully amorphous material, characterized by a glass transition temperature $T_{g}$ measured at $6.4{ }^{\circ} \mathrm{C}$ by differential scanning calorimetry, is obtained and showed a thick oily-like state. This low glass transition helps in the formation of spherical organic nanoparticles upon nanoprecipitation in water to minimize surface tension effects and prevent recrystallization in the course of time that could impart FONs with undesirable aggregation effects or various crystalline phases. ${ }^{[25]} \mathrm{FON}$ nanoprecipitation typically proceeds at room temperature from the quick addition and mixing of a concentrated dye solution in tetrahydrofuran (THF) within a larger volume of water using magnetic stirring, to cause efficient dispersion (Figure 2A).
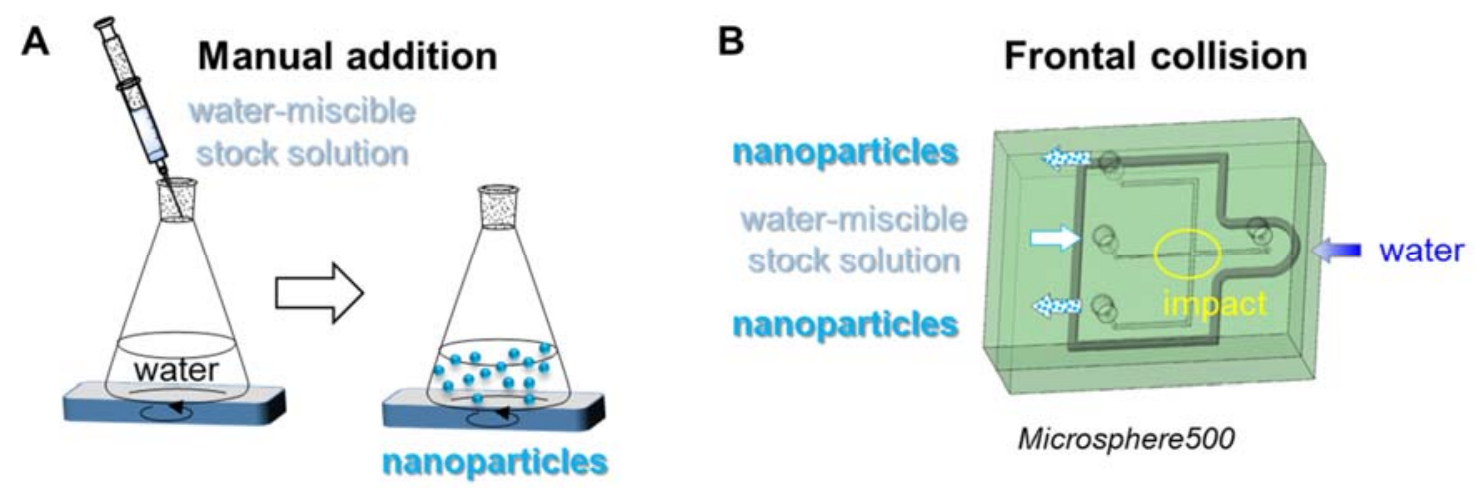

Figure 2. FON fabrication following flash precipitation. A) Quick manual addition of a stock fluorophore solution into a large volume of water under vortex stirring. B) Transparent 3D-view of the Microsphere500 micromixer allowing for high-impact frontal collision of two opposite flows, containing respectively the stock solution (channel with a $300 \mu \mathrm{m} \times 300 \mu \mathrm{m}$ square section) and water (channel with a $600 \mu \mathrm{m} \times 600 \mu \mathrm{m}$ square section). The thicker gray line features the rubber seal, sandwiched between the two aluminum plates (green), to avoid leakage 
due to high-pressure injection, and the thinner line corresponds to the microfluidic circuit itself, etched in the bottom plate. All tubing inlets and outlets are fixed on the top plate and indicated by a circular aperture.

Controlling two main criteria like the concentration of the organic stock solution and the mixing rate thus appears crucial. In order to independently investigate their influence in a defined manner, we resorted to the Microsphere500 mixing chamber comprising one channel $(600 \mu \mathrm{m}$ large section), supplied with water and slightly up-shifted with regard to an opposite smaller channel (300 $\mu \mathrm{m}$-large section), funneling the organic stock solution (Figure $2 \mathrm{~B})$. On the basis of previous studies involving manual injection in water of a $0.1 \mathrm{wt} . \%$ dye solution in THF, we considered an initial $0.5 \mathrm{wt} . \%$ dye concentration as a median value and varied this mass fraction from 0.025 up to $1 \mathrm{wt} . \%$. The mixing rate was indirectly tuned by changing independently the flow rates of each channel and the respective flow rate ratio, which controls the mass ratio. We thus carried out two distinct experiments $\mathbf{A}$ and $\mathbf{B}$, corresponding to the conditions detailed in Table S1, namely variable organic solution (o) and water (w) flow rates (constant flow rate ratio (o:w) and stock solution concentration), and variable stock solution concentration (constant (o) and (w) flow rates, and flow rate ratio (o:w)) respectively. For each experiment $\mathbf{A}$ and $\mathbf{B}$, the samples were collected $4 \mathrm{~s}$ after starting the mixing process to avoid composition inhomogeneity. FON size distribution was measured by dynamic light scattering (DLS) right after the sample collection. For experiment A, the FON Z-average size (diameter) $D_{Z}$ decreased from 193 to 86 $\mathrm{nm}$ (with a polydispersity index (PDI) averaged over the five measurements found around 0.215 ) when the flow rate increased by a factor of 5 (Figure $3 \mathrm{~A}$ ). In the same way, the $D z$ values for experiment $\mathbf{B}$ decreased from 166 to $66 \mathrm{~nm}$ (with a similar averaged PDI around 0.219) along with the reduction of the injected stock organic solution (Figure 3B). Such evolution recalls 
previous results obtained with a $3 \mathrm{D}$ flow focusing chamber although in the latter case, control of the size polydispersity was delicate to be achieved with high flow focusing ratios. ${ }^{[26]}$. By contrast to $D_{Z}$ hydrodynamic diameters, the dry diameter $D_{T E M}$ values, determined from statistical numbering of TEM-imaged FONs for samples issued from experiment B (Figure 5E) were found quite constant at around 61-66 nm with a relatively narrow size dispersion (Table S2 and Figure S2). Such large distinct evolutions may appear quite puzzling at first sight and could suggest aggregation at higher concentration to explain the size increase, which appears quite incompatible with the well-defined nanoparticles observed by TEM or later on by AFM. As mentioned above, compound ALD displays quite a low $T_{g}$ below room temperature. We suspect fragmentation in the TEM chamber of the honey-like FONs into smaller nanoparticles with more stable geometry, hence very similar size distributions are obtained.

A

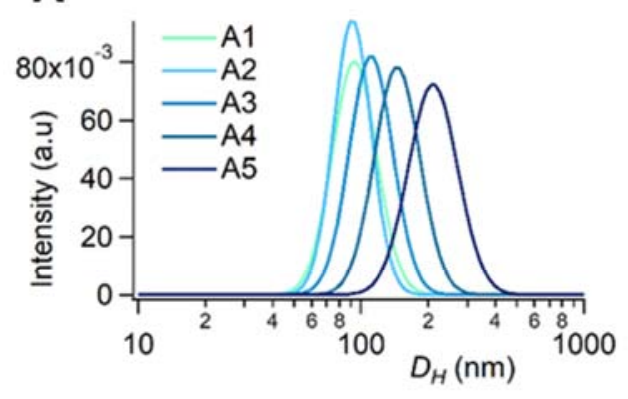

B

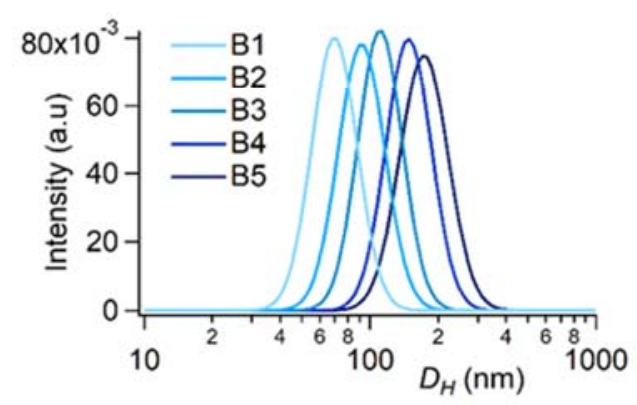

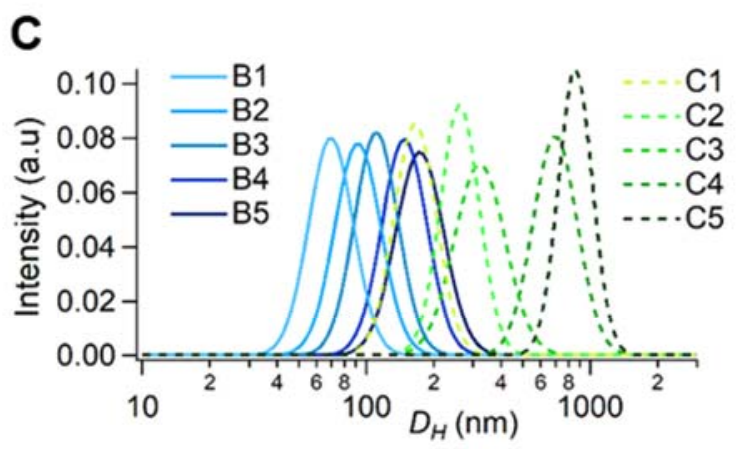

D

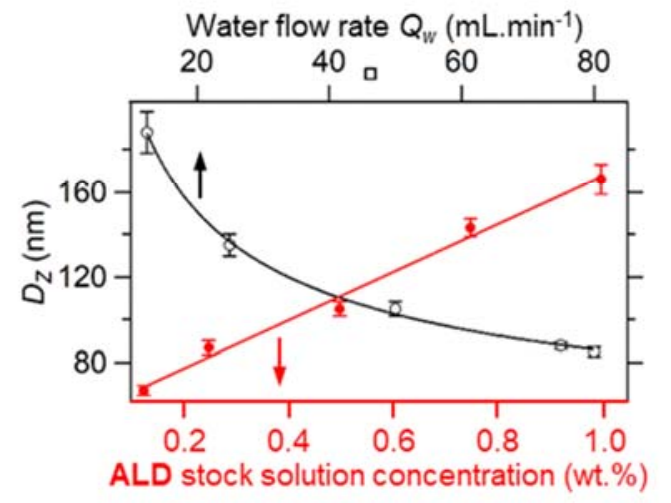



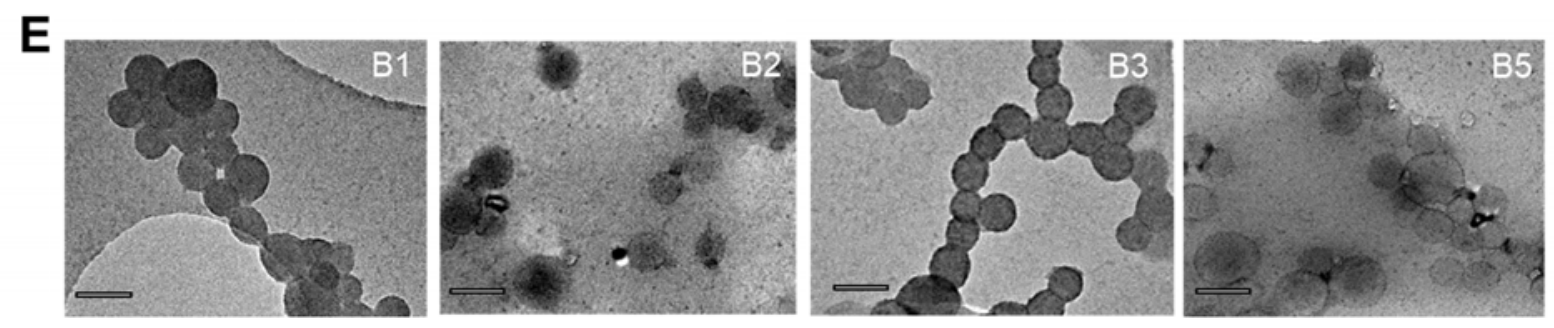

Figure 3. DLS measurements of FON dispersions made of ALD fluorophores and obtained by means of the Microsphere500 mixing chamber: A) experiment A, B) experiment B, and by means of vortex stirring: C) experiment C. D) Variation of the Z-average size (diameter) $D Z$ of FONs as a function of the water flow rate $Q_{w}$ (experiment $\mathbf{A}$ ) and stock solution concentration (experiment B); error bars were calculated using a 95\% confidence interval. E) TEM imaging of FONs issued from experiment B (sample B4, not represented here, gave intractable images). Scale bar: 100 nm.

As for the $D z$ values, , it was interesting to find that they decreased as a function of the water flow rate $Q_{w}$, following a power law $D_{Z}=544 \mathrm{Q}_{\mathrm{w}}{ }^{-0.424}$, as commonly reported for micromixers where one flow rate predominates, even in the case of systems with distinct geometry or smaller flow velocity. ${ }^{[27]}$ By contrast, the $D_{Z}$ values evolve quite linearly as a function of the stock solution concentration (Figure 3D). Hence, we can suggest that high flow rates favor efficient dispersion of the organic constituents and nucleation upon supersaturation, conducting to smaller nanoparticles with a narrow size distribution. Transmission electron microscopy imaging showed indeed smooth and well spherical nanoparticles whose size appears very homogeneous. Interestingly, compared to identical final ALD concentrations (experiment B), nanoparticles obtained upon manual addition in water of ALD solutions with a distinct stock solution concentration (experiment C) systematically showed larger mean size and size polydispersity (averaged PDI equal to 0.230) (Figure 3C, Table S1). Finally, we tested the 
reproducibility of experiment $\mathbf{A}$ where flow impact rather than stock solution concentration exerts higher influence on FON size. We found that the diameter evolution of the second series differs by only $5 \%$ compared to the first one. All these elements let us propose that FON fabrication using the Microsphere500 mixing chamber yields high-quality and reproducible samples.

Size measurements were completed with surface potential $\zeta$ measurements by means of zetametry to investigate FON colloidal stability in water. Surprisingly, largely $\zeta$ negative values of $-39 \pm 8 \mathrm{mV}$ were obtained. Given the FON size, $\zeta$ values larger than $-30 \mathrm{mV}$, indicate great colloidal stability in water which was indeed found over one year at least (Figure S3). This apparently surprising result, despite the large ALD hydrophobic character and absence of ionizable units, has already been observed and ascribed to the adsorption at the FON surface of traces of fatty acids, present as contaminants. ${ }^{[28]}$

To conclude on this part, it is worth noting that the rate of the impacting organic flow used in our case is significantly reduced by a factor of 4 to 5 comparatively to the CIJ and MVIM mixer geometries used in literature, while nanoparticles with size of similar Z-average $\left(D_{Z} \approx 60\right.$ $200 \mathrm{~nm}$ ) are obtained. ${ }^{10 \mathrm{~b}}$ This comparison is based on literature data since we could not benefit from direct assay on the last two mixers. This lets us propose that the Microsphere500 mixer, allying ultrafast opposite impacting flows, vortex mixing and easy modulation of the kinetic energy, would represent an attractive setup to significantly limit the consumption of precious organic materials in the formulation of active nanoparticles.

\subsection{Evolution of the FON photophysical properties as a function of the mixing rate}

Since fabricated FONs in these studies are devoid of any additional surfactant or polymer, direct assessment of their photophysical properties as a function of their intrinsic size and method of 
preparation can be performed. All nanoparticle dispersions displayed bright bluish-green emission, centered at $500 \mathrm{~nm}$ upon excitation in the UV absorption band composed of a shoulder at $375 \mathrm{~nm}$ and a maximum at $345 \mathrm{~nm}$ that are related to charge transfer from the triphenylamino center to the formyl and biphenyl moieties, respectively. ${ }^{[23]}$ Interestingly, this emission maximum is quite similar to that reported for non-doped thin films fabricated by spin-coating a $1 \mathrm{wt} \%$ dye solution in chloroform and subsequently vacuum-dried, ${ }^{[23]}$ thereby indicating that local polarity effects originate from the dyes themselves, independently of the surrounding hydration shell. The constant energy value for the emission signal of all nanoparticles lets us suggest quite similar dye spatial arrangements from one FON batch to another since any significant difference in local surroundings would cause notable energy shift of the transition. At this step, performing fluorescence microscopy imaging on single nanoparticles could be very stimulating to infer their proper responses, but such experiments represent an entire filed of investigations per se, beyond the scope of the current studies. However, particular attention paid to the emission intensity and dynamic relaxation of the excited state revealed significant difference between the fabrication methods A and B. Each sample was diluted with Millipore water in the same amount before measurements to limit inner filter effects, especially in excitation. In agreement with the constant concentration of fluorescent material in all samples of experiment $\mathbf{A}$, the absorption and emission spectra display almost the same intensity, except for sample A5. The latter contains larger FONs formed under larger flow rates, and show significant contribution of light scattering (Figure 4A). At this step, we must admit that we have no reasonable explanation to support the observed bathochromic and hypochromic shifts. Formation of $\pi-\pi$ aggregates that is commonly evoked is indeed contradictory with the almost constant value of the fluorescence quantum yields $\Phi_{f}$ measured to be 0.16 on average. As for the samples of experiment $\mathbf{B}$ where the concentration of 
injected fluorophores is twice as high from one sample to another, the intensity of the absorption and emission spectra logically increased proportionally (Figure 4B). No change in the general shape occurred, hence intermolecular interactions within the nanoparticles are similar between the different batches. The fluorescence quantum yield $\Phi_{\mathrm{f}}$ was found very close to that for samples A, namely at 0.17 on average. Since ALD fluorophores, characterized by strong charge transfer in their radiative transitions, ${ }^{[23]}$ are very sensitive to local polarity effects in their excited states, any strong structural changes in the fluorophore surroundings would be accompanied by notable modification of their emission energy and intensity as well. The constant value found for $\Phi_{\mathrm{f}}$ tends to indicate similar dye self-assembling within FONs whatever the flow energy impact. 
A
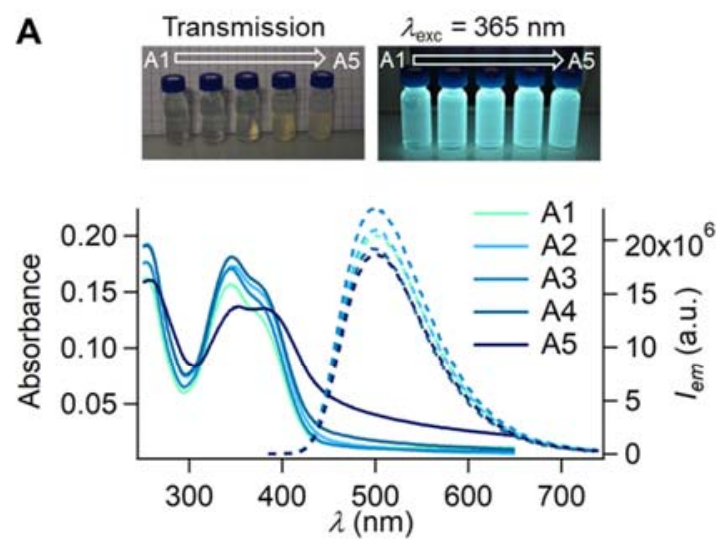

C

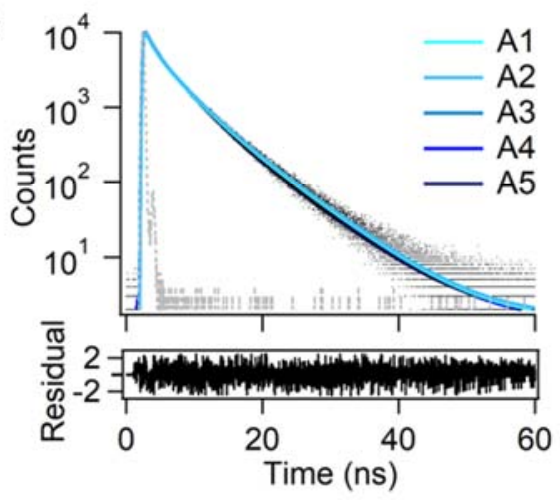

D

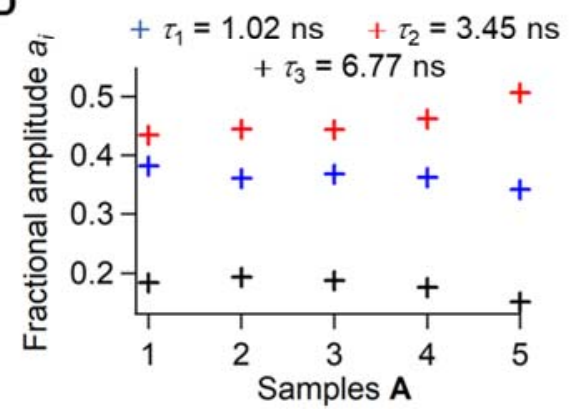

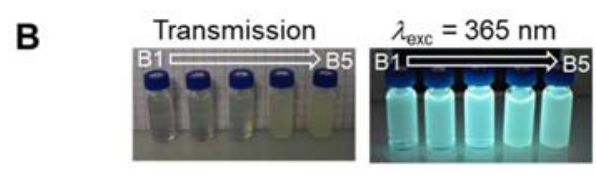
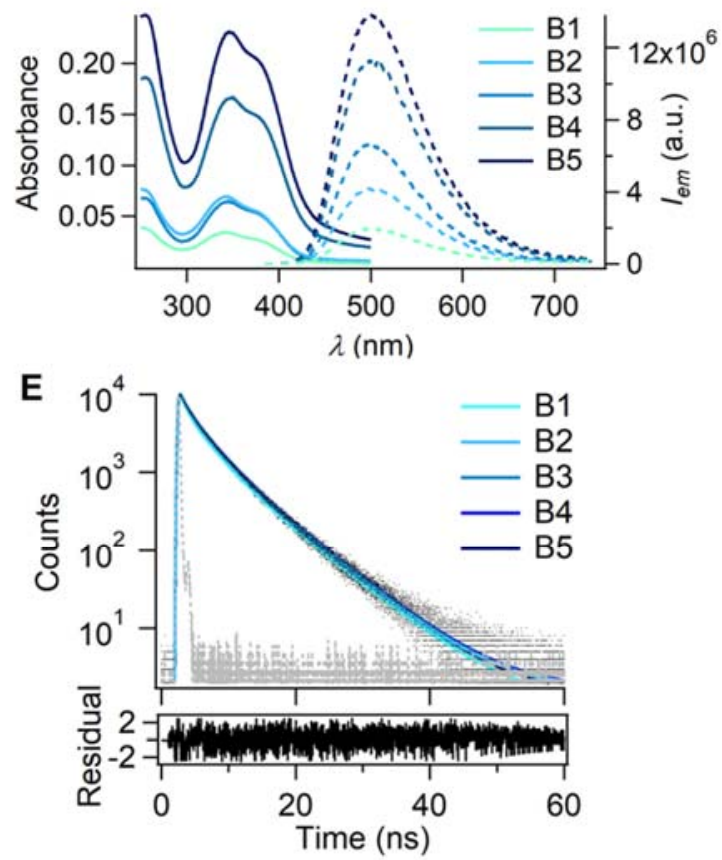

$\mathbf{F}$

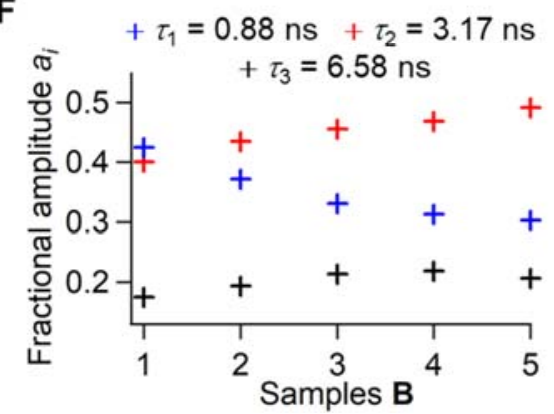

Figure 4. ALD FON dispersions under ambient light and $365 \mathrm{~nm}$ excitation (top) and UV-vis absorption and emission spectra $\left(\lambda_{\mathrm{exc}}=375 \mathrm{~nm}\right)$ (bottom) of aqueous ALD FON dispersions: A) issued from experiment A, B) issued from experiment $\mathbf{B}$. Fluorescence time decays (middle) $\left(\lambda_{\mathrm{exc}}\right.$ $=340 \mathrm{~nm}, \lambda_{\mathrm{em}}=501 \mathrm{~nm}$ ) and evolution of the fractional amplitudes $a_{i}$ after reconvolution from the excitation pulse response (gray trace) and global fitting following a three-exponential mathematical law (bottom) of aqueous ALD FON dispersions issued from: C-D) experiment A, 
and E-F) experiment $\mathbf{B}$ (residual traces of the global fitting are indicated on the bottom of the lifetime decays in Figures C and E).

Yet, accurate examination of the FON fluorescence decays revealed subtle evolutions with the FON diameter. The decays, obtained after excitation at $340 \mathrm{~nm}$ and recording the emission signal at $501 \mathrm{~nm}$, required a multi-exponential fit and provided three distinct lifetimes using global fitting analyses $\left(\tau_{1}=0.88(\mathbf{A}), 1.02(\mathbf{B}) \mathrm{ns} ; \tau_{2}=3.17(\mathbf{A}), 3.45(\mathbf{B}) \mathrm{ns} ; \tau_{3}=6.58(\mathbf{A}), 6.77(\mathbf{B}) \mathrm{ns}\right)$ for the samples of both experiments, recalling previous behaviors investigated in solid thin film (Figure 4C-D). The smaller lifetimes found overall for the samples A compared to those of samples B may be related to the slightly distinct surroundings in terms of water:THF composition in the final mixture since the injected THF volume increased from sample B1 through sample B5. As for the evolution of the lifetime fractional amplitudes $a_{\mathrm{i}}$ along with the FON diameter, two opposite evolutions could clearly be observed (Figure 4E). The $a_{2}$ contribution of the intermediate lifetime $\tau_{2}$ globally increased with the FON size. Conversely, the $a_{1}$ component of the shorter lifetime $\tau_{1}$ decreased, even more neatly for samples B. Based on previous description of FONs in water, molecules at the interface undergo significant quenching in contact with water due to vibrational relaxations and would be representative of the shorter lifetime $\tau_{1 .}{ }^{[29]}$ On the contrary, the intermediate lifetime $\tau_{2}$, whose contribution dominates for fluorophores processed as neat thin films, would suggest a bulk origin. So, observing a raise of the shorter lifetime component $\tau_{1}$ and a drop of the intermediate lifetime component $\tau_{2}$ with the FON size reduction agrees with an increase in the surface-to-volume ratio, showing the sensitivity of ALD fluorophores toward their close surrounding. ${ }^{[30]}$ We thus harnessed such sensitivity to show that bicomposite FONs can also be obtained using the high-impact Microsphere500 microfluidic 
chamber with little risks of demixing into separated components caused by high dye concentrations in stock solutions.

\subsection{Bicomposite FONs with tunable color emission and amplified emission intensity as a result of dye coprecipitation}

To this aim, we targeted the generation of bicomposite FONs out of energy donors and acceptors, which can promote efficient energy transfer for spatially confined fluorophores. We thus employed the red-emissive fluorophore VIN, displaying large structural similarity with the bluish-green fluorophore ALD and yielding amorphous material $\left(T_{g}=11.9^{\circ} \mathrm{C}\right)$, so that high mixing entropy is favored in the final bicomposite FONs (Figure 1). Its absorption spectrum broadly overlaps the emission spectrum of the bluish-green emitter, yielding efficient Förster resonance energy transfer (FRET), with a Förster radius $\mathrm{R}_{0}$ valued to be $39 \AA$ in tetrahydrofuran from calculations. To validate our approach, we prepared four stock solutions in THF with a varying ALD:VIN composition: 100:0, 95:5, 80:20 and 0:100 \%. We fixed the flow rates of the microchannels funneling water and THF solution respectively at 50 and $2 \mathrm{~mL} \cdot \mathrm{min}^{-1}$, and ALD:VIN solutions at a 0.25 wt. \% total dye concentration, which represents a median value among the previous assays. All samples investigated by TEM formed well-defined nanoparticles, the VIN FONs being a little less defined (Figure 5C). Their zeta potential values $\zeta$ were found to be around $-42 \pm 4 \mathrm{mV}$, supporting again large colloidal stability in water. Lower diameters could be noticed for all batches compared to the size distribution measured for experiment B2 involving a similar dye concentration. This is likely to be due to a dilution effect, slightly higher than expected during solution preparation, thereby generating smaller FONs. Nevertheless, this does not question the logic size evolution obtained through a series of samples, as demonstrated by the above reported reproducibility assay, provided that FON are generated in the row, using the same 
tubing, solvents and plastic syringes as experimented here. It is worth noting that the FON diameter varied despite the constant amount of injected organic material, which can be ascribed to distinct dipolar interactions established between the fluorophores and water molecules (Figure $5 B)$.
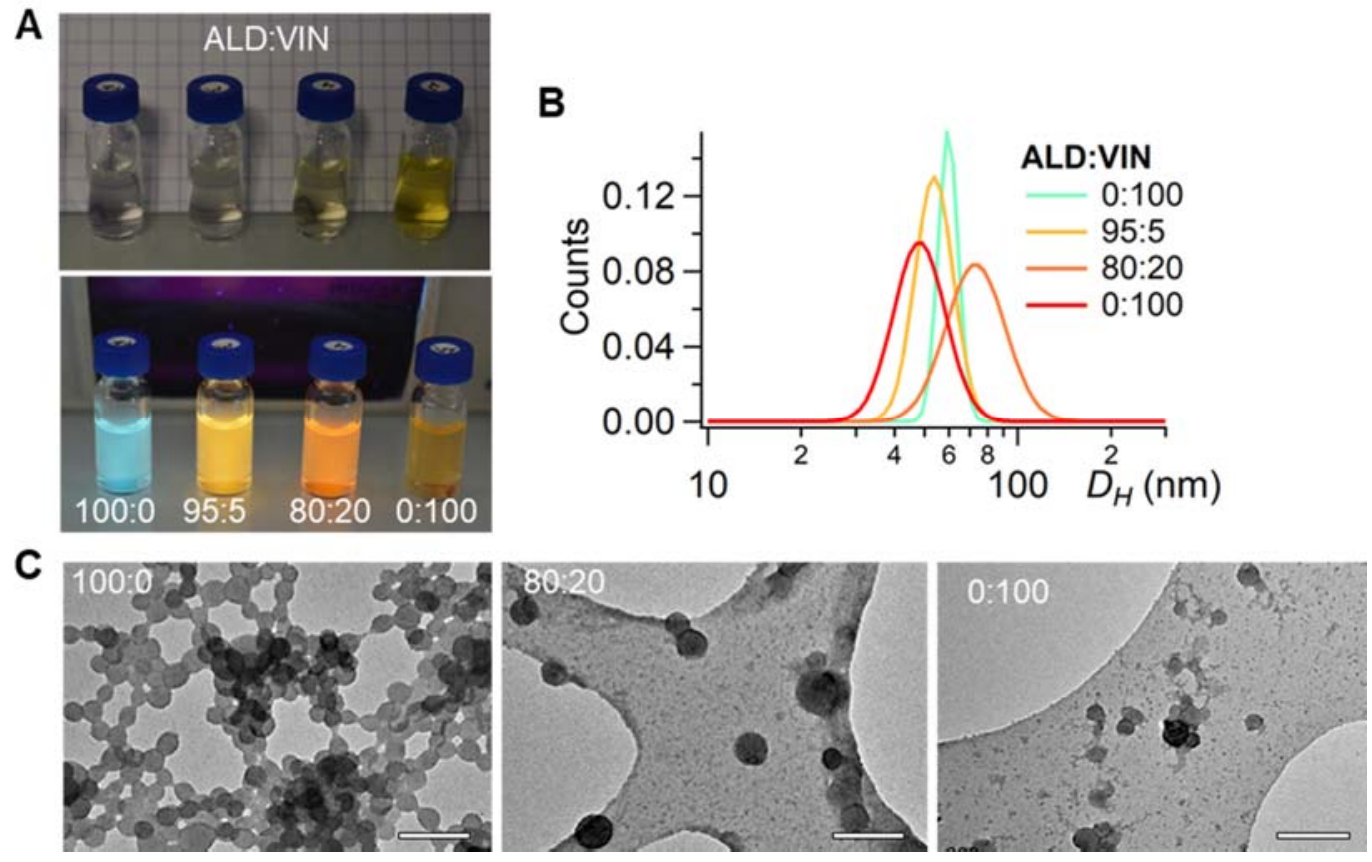

Figure 5. ALD:VIN FON dispersions obtained from the Microsphere 500 mixing chamber for distinct compositions 100:0, 95:5, 80:20 and 0:100. A) Vials in transmission (top) and under 365 nm excitation (bottom). B) DLS distributions. C) TEM imaging using holey carbon-coated copper grids (sample 95:5 was not imaged for priority reasons). Scale bar: $200 \mathrm{~nm}$.

In agreement with the increasing amount of compound VIN, an absorption band centered at $452 \mathrm{~nm}$ rose (Figure 6A), typical of the lower charge transfer excited state calculated from TDDFT computations. ${ }^{[23]}$ Given the absence of spectral overlap in the visible between the absorption spectra of the ALD blue and VIN red emitters and the match at $375 \mathrm{~nm}$ between a maximal absorption for ALD and a minimal absorption for VIN, the emission spectra were 
recorded upon excitation at $375 \mathrm{~nm}$ and $452 \mathrm{~nm}$ to distinguish the contribution of both dyes and assess the extent of energy transfer. For $\lambda_{\mathrm{exc}}=375 \mathrm{~nm}$, the emission band centered at $500 \mathrm{~nm}$ was typical of pure FON $\mathrm{ALD}$ and dramatically shifted to $589 \mathrm{~nm}$ for FONs comprising only a 5 mol. $\%$ amount of VIN (Figure 6B), yielding bright sun yellow emission (Figure 6A).
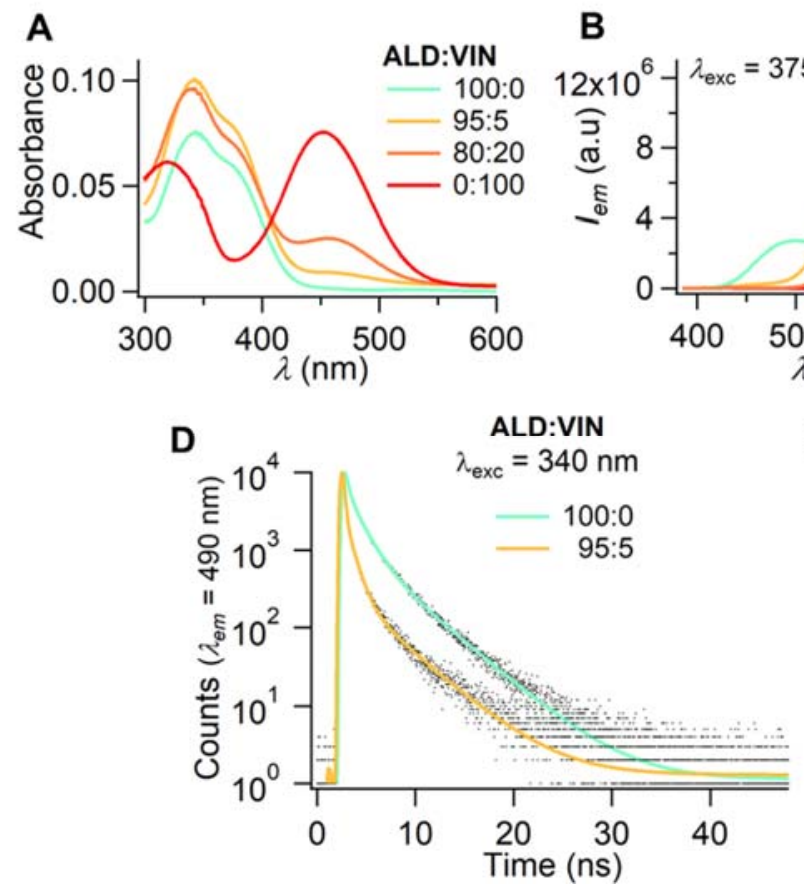

B

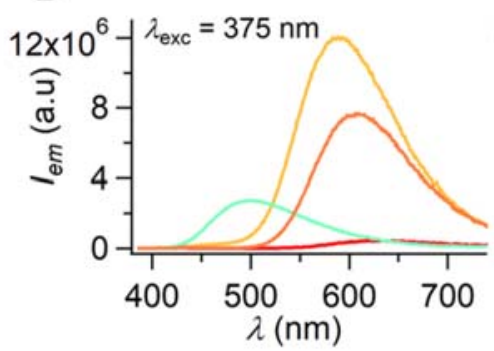

C

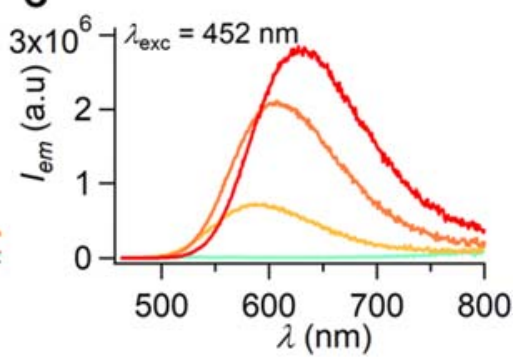

E

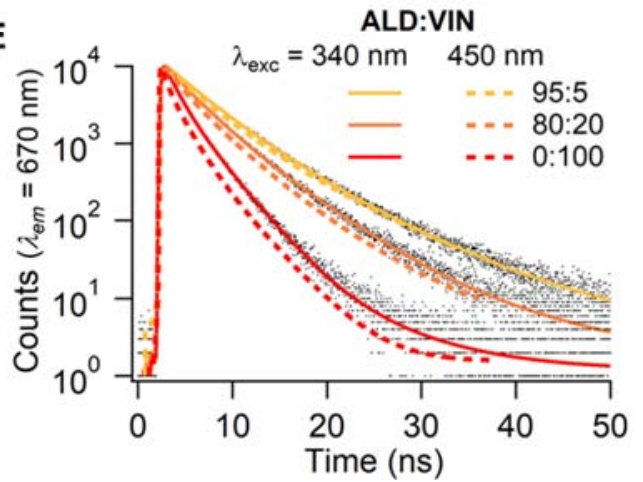

Figure 6. Aqueous ALD:VIN FON dispersions obtained using the Microsphere500 mixing chamber with carrying composition 100:0, 95:5, 80:20 and 0:100: A) UV-vis absorption spectra, B) emission spectra upon excitation at $\lambda_{\mathrm{exc}}=375 \mathrm{~nm}$ where mainly ALD absorbs, C) emission spectra upon excitation where mainly VIN absorbs, D) Fluorescence time decays upon excitation at $\lambda_{\mathrm{exc}}=340 \mathrm{~nm}$ and recording at $\lambda_{\mathrm{em}}=490 \mathrm{~nm}$ where ALD exclusively emits light, E) fluorescence time decays upon excitation at $\lambda_{\mathrm{exc}}=340$ and $450 \mathrm{~nm}$ and recording at $\lambda_{\mathrm{em}}=670$ nm where VIN exclusively emits light.

Its intensity appeared to be 17 times higher compared to that obtained upon direct excitation at $452 \mathrm{~nm}$ and 700 times higher if VIN alone were excited at the same concentration (Figure 6C). 
From the donor emission spectra, the energy transfer quantum efficiency $\Phi_{\text {FRET }}$ from ALD to VIN could be assessed to be 74 and $96 \%$ for FON compositions of 95:5 and 80:20 respectively. This large value could be confirmed through time-resolved fluorescence measurements, by exciting at two distinct wavelengths, namely 340 and $450 \mathrm{~nm}$, and recording the fluorescence decays at 490 (Figure 6D) $\mathrm{nm}$ or $670 \mathrm{~nm}$ (Figure 6E). A strong decay of the blue-greenish signal was again noted for the 95:5 FON composition where the amplitude-averaged lifetime $\langle\tau\rangle_{\mathrm{a}}$ indeed decreased by $75 \%$ from 0.81 to $0.23 \mathrm{~ns}$. Very interestingly, the fluorescence decay at 670 $\mathrm{nm}$ shortened ( $\langle\tau\rangle_{\mathrm{a}}$ dropped from 4.46 to $\left.1.45 \mathrm{~ns}\right)$ for increasing amounts of red emitter VIN, whatever the excitation at 340 or $450 \mathrm{~nm}$, whereas similar time constants would have been expected (Table 1). Unfortunately, we were not allowed to distinguish any negative time contribution in the fluorescence decay of VIN upon exciting at $340 \mathrm{~nm}$, which features very efficient energy transfer from ALD to VIN molecules, namely less than a dozen of ps, since the instrumental response function of the acquisition card is not higher than 120 ps. This trend is actually to be related to distinct polar surroundings undergone by the red dyes as a function of the FON composition. This is illustrated by the progressive bathochromic shift $(589,607$, and $630 \mathrm{~nm}$ ) of the emission band undergone for larger amounts (5, 20 and $100 \%)$ of VIN whose dipole moment was calculated to be 9.68 D against 7.93 D for ALD. Since VIN is strongly solvatochromic and known to be significantly quenched in polar surroundings (water included) upon strong twist in the excited state, ${ }^{[31]}$ color tuning is here caused by the change in polarity ${ }^{[32]}$ around the red emitters with the composition, rather than by overlapping emission signals. ${ }^{[21 c]}$ Nanoprecipitation of pure ALD or FVIN FONs out of fluorophore mixes could be ruled out on the basis of the above calculated Förster radius value, which is not compatible with the FON 
diameter, larger than $40 \mathrm{~nm}$, and the almost quantitative energy transfer observed for only a $5 \%$ amount of FVIN energy acceptors with regard to the ALD energy donors.

Table 1. Size distributions and photophysical properties of color-tunable FON dispersions made of ADL:VIN of varying composition.

\begin{tabular}{|c|c|c|c|c|c|c|c|}
\hline \multirow{2}{*}{$\begin{array}{c}\text { FON } \\
\text { ALD:VIN }\end{array}$} & \multirow{2}{*}{$\begin{array}{c}D_{Z} \\
(\mathrm{~nm}) \\
(\mathrm{PDI})\end{array}$} & \multirow{2}{*}{$\begin{array}{c}\lambda \max (a b s) \\
(\mathrm{nm})\end{array}$} & \multirow{2}{*}{$\begin{array}{c}\lambda_{\max }(\mathrm{em}) \\
(\mathrm{nm})^{[\mathrm{a}]}\end{array}$} & \multirow{2}{*}{$\Phi_{\mathrm{f}}^{[\mathrm{b}]}$} & \multicolumn{3}{|c|}{$\langle\tau\rangle_{\mathrm{a}}{ }_{\mathrm{a}}^{[\mathrm{c}]}(\mathrm{ns})$} \\
\hline & & & & & $\begin{array}{l}\lambda_{\mathrm{exc}}=340 \mathrm{~nm} \\
\lambda_{\mathrm{em}}=490 \mathrm{~nm}\end{array}$ & $\begin{array}{l}\lambda_{\mathrm{exc}}=340 \mathrm{~nm} \\
\lambda_{\mathrm{em}}=670 \mathrm{~nm}\end{array}$ & $\begin{array}{l}\lambda_{\mathrm{exc}}=340 \mathrm{~nm} \\
\lambda_{\mathrm{em}}=670 \mathrm{~nm}\end{array}$ \\
\hline 100:0 & $\begin{array}{c}61 \\
(0.019)\end{array}$ & 375,342 & 500 & 0.13 & 0.93 & - & - \\
\hline $95: 5$ & $\begin{array}{c}52 \\
(0.069)\end{array}$ & $\begin{array}{c}452, \\
375,342\end{array}$ & 589 & 0.34 & 0.23 & 4.54 & 4.46 \\
\hline $80: 20$ & $\begin{array}{c}70 \\
(0.250)\end{array}$ & $\begin{array}{c}456, \\
375,340\end{array}$ & 607 & 0.22 & - & 3.48 & 3.04 \\
\hline 100:0 & $\begin{array}{c}44 \\
(0.142)\end{array}$ & 452,320 & 630 & 0.10 & - & 1.93 & 1.45 \\
\hline
\end{tabular}

[a] Excitation indifferently at 375 or $452 \mathrm{~nm}$ (except for neat ALD FONs). [b] Valued using coumarin $540 \mathrm{~A}$ as a fluorescence standard $\left(\Phi_{\mathrm{f}}=0.38\right)$. [c] Fluorescence decays were modeled through a triexponential law:

$$
I(t)=\sum_{i=1}^{3} \alpha_{i} \exp \left(-t / \tau_{i}\right) \quad \text { with the fractional amplitudes } \quad a_{i}=\alpha_{i} / \sum_{j} \alpha_{j} \quad\langle\tau\rangle_{a}=\sum_{i=1}^{3} a_{i} \tau_{i}
$$

Comparative photophysical experiments performed on mixtures of pristine ALD FONs and VIN FONs following the same ALD:VIN ratios than those reported for FON blends indeed evidenced the absence of significant energy red shift upon exciting ALD FONs for a 95:5 ALD:VIN ratio (Table S3 - Figure S4). Only the 80:20 ALD:VIN composition may suggest slight interactions between the separated FONs as a consequence of favorable hydrophobic 
interactions rather than any interactions between solvated individual dyes whose photophysical properties remain unchanged (Figure S5). Indeed, partial energy shift of the ALD emission band that broadens due to the VIN contribution and possible inner filter effects was observed (Table S3 - Figure S4B-C and associated comments), while the amplitude-averaged lifetime $\langle\tau\rangle_{a}$ of the ALD units was reduced by half (Figure S6). However, in that case, energy transfer that would then operate at the interface between ALD and VIN FONs is by no way comparable to that observed for bicomposite FONs. All these observations thus support the fact that FRET operates within the bicomposite FONs as a result of ALD and VIN coprecipitation within the same nanoparticles that do not demix into separate FONs. Modeling the kinetics of such an energy process in FONs as a function of the amount of energy acceptors with respect to that of the energy donors would require more complex approaches, taking into account energy migration through space and through bond, namely through dipole-dipole interactions or hopping, as described in literature. ${ }^{[33]}$

To conclude on this part, diluting red-emissive dyes in hydrophobic matrices, serving as energy antenna, appears as a very attractive and ubiquitous approach to generate bright far-red FONs, thereby avoiding competitive radiationless deactivation of low-energy emitters in polar surroundings. Such observation echoes the recent theoretical developments on fluorescence quenching in solution and fluorescence recovery in AIEgene nanoparticles. Such studies point out the dramatic energy shift of the conical intersection (CI) between the $\mathrm{S}_{1}$ excited state and $\mathrm{S}_{0}$ ground state undergone along the potential energy surface for self-assembled fluorophores, thereby reducing the radiationless deactivation pathway. ${ }^{[12 b]}$ Based on previous transient absorption spectroscopy experiments carried out on a similar compound, we herein suspect that such CI shift may operate to explain the considerable dye emission amplification observed for 
VIN fluorophores embedded in ALD FONs compared to dyes dissolved in THF (Figure S5) or self-assembled in "monocompound" FONs, which would result not from steric effects but from strongly exacerbated polarity effects. To go a step further and harness the high spatial confinement of dyes in organic nanoparticles, we finally tested the ability of photocrosslinkable fluorophores like ALD to be reactive after being processed as FONs, which to our knowledge as never been explored so far, despite the great interest in the generation of photopolymerized functional nanoarchitectures. ${ }^{[34],[35]}$

\subsection{Photoreaction performed in pre-formed FONs}

We here based our approach on the fact that ALD, processed as thin films, could undergo solidstate through a photoinitiated radical polymerization of its acrylate units. ${ }^{[23]}$ In order to limit the action of oxygen acting as a radical scavenger, we privileged the well-documented thiol-ene reaction, highly compatible with water and ambient air and temperature conditions. ${ }^{[36]}$ Pentaerythritoltetrakis(3-mercaptoprioionate) (PT-SH), containing four thiol units, was chosen as a crosslinker while 2,4-6-trimethylbenzoyl-diphenylphosphine (TPO) was retained as a hydrophobic photoinitiator (Figure 1). The latter choice was motivated by previous results showing high deactivation of TPO photodissociation upon competitive energy transfer toward low-energy lying red emitters like VIN. ${ }^{[24]}$ Thanks to the aforementioned results emphasizing amplified energy transfer in FONs, we thus privileged the use of ALD instead of VIN, despite a TPO excitation in the UV range, namely at $365 \mathrm{~nm}$, overlapping the ALD absorption spectrum. Based on preliminary assays performed on thin films to obtain efficient crosslinking within a reasonable irradiation time, two distinct PT-SH:ALD composition with a molar ratio of $0.1: 1$ and 0.5:1 (FON $\mathrm{FO}_{0.1}$ and $\mathrm{FON}_{0.5}$ ), corresponding respectively to a $20 \%$ and equimolar content of thiol units compared to the acrylate ones, were tested while keeping constant at $2.5 \mathrm{~mol} . \%$ the amount 
of TPO photoinitiator with regard to ALD. FONs devoid of TPO and PT-SH were also prepared to serve as control samples $\left(\mathrm{FON}_{0}\right)$.

From the absorption and emission spectra before photoirradiation, the shape and intensity maxima of the bands were found to be almost the same, given the experimental uncertainty upon dilution or precipitation in water, showing weak impact of the additional components like PT-SH known to promote fluorescence deactivation upon reductive electron transfer (Figure 7B and 7D). ${ }^{[37]}$ After photoirradiation in deoxygenated conditions, noticeable changes in absorption could be observed, especially for FON 0.5 with a higher PT-SH content, where the low-energy shoulder at $375 \mathrm{~nm}$, ascribed to the charge transfer excited state responsible for emission, is reduced in intensity (Figure 7C).

A
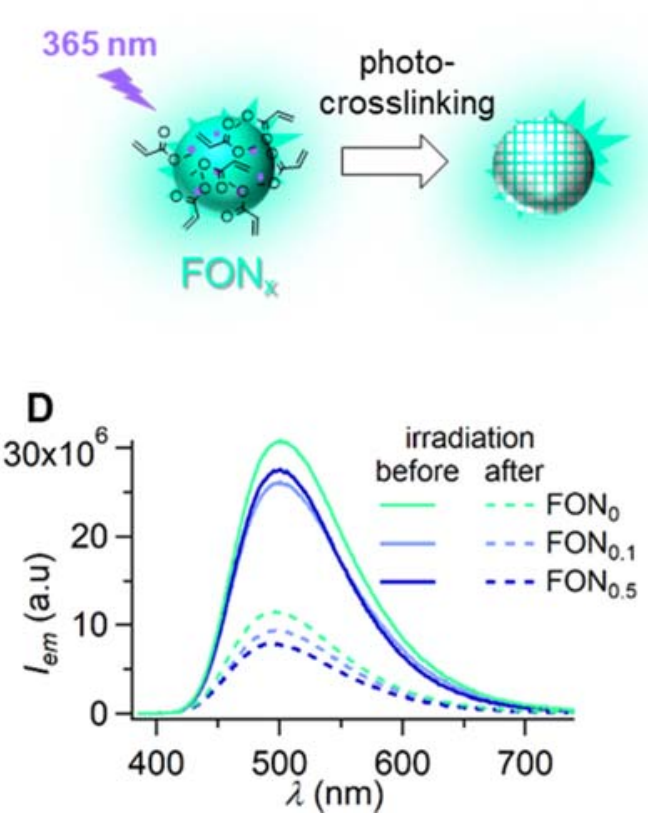

B
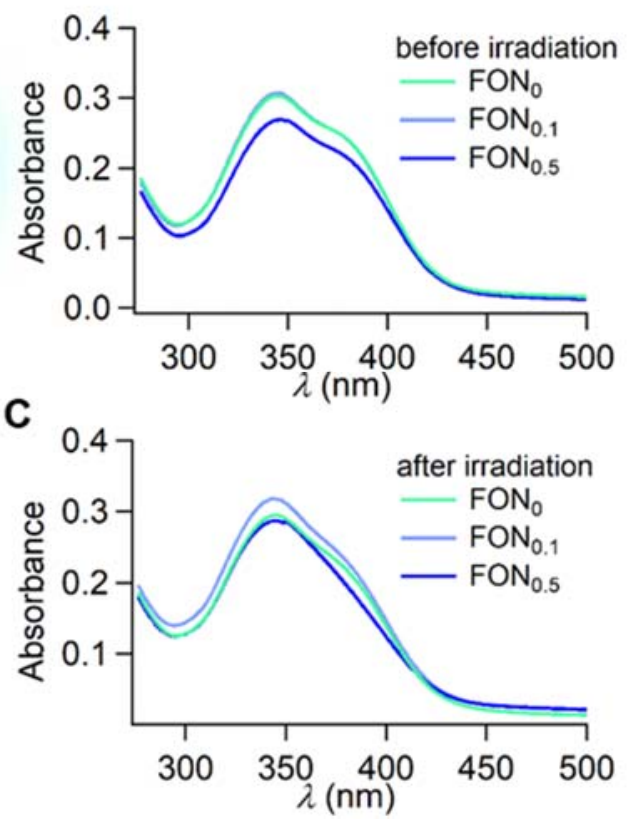

Figure 7. A) Schematic description of photocrosslinking of FONs made of TPO:PT-SHx:ALD upon irradiation at $365 \mathrm{~nm}$ for $5 \mathrm{~min}$. $\left(30 \mathrm{~mW} \cdot \mathrm{cm}^{-2}\right)$. UV-vis absorption spectra: B) before irradiation, C) after irradiation, and D) emission spectra $\left(\lambda_{e x c}=375 \mathrm{~nm}\right)$ of FONs with varying 
molar compositions of PT-SH (ALD alone: $\mathrm{x}=0$; TPO:0.1 PT-SH:ALD $\mathrm{x}=0.1$; TPO:0.5 PTSH:ALD $\mathrm{x}=0.5$ ) where TPO is employed at a 2.5 mol. \% ratio with respect to ALD. Irradiation was performed on deoxygenated FON dispersions by prior argon bubbling.

Particular focus on control FON $\mathrm{F}_{0}$ made exclusively of ALD showed a similar reduction, albeit much less pronounced than for any other composition (Table 1). When looking at the emission spectra, all samples remained very emissive, even though the signal intensity appeared to be about $30-36 \%$ of the initial value. Again, the most significant reduction is noticed for FON 0.5 containing TPO and the higher amount of PT-SH crosslinker. We thus here suspect that photoredox processes assisted by UV irradiation or electron transfer from the thiol units, rather than the photocrosslinking reaction itself, are responsible for the photodegradation of the fluorophores promoted in their excited state. Indeed, photoirradiation of the FON dispersions in the absence of prior argon bubbling caused dramatic loss of the emission intensities to $6-16 \%$ of their initial values.

In order to prove the reality of photocrosslinking, structural nanometer scale investigations were carried out first by resorting to TEM imaging. While the aspect of FON 0 and $\mathrm{FON}_{0.5}$ appeared smooth and round before irradiation, it systematically evolved to a more grainy structure while keeping its overall spherical shape, which lets us again suggest homogeneous composition of the precipitated composite FONs during fabrication (Figure 8). 
A

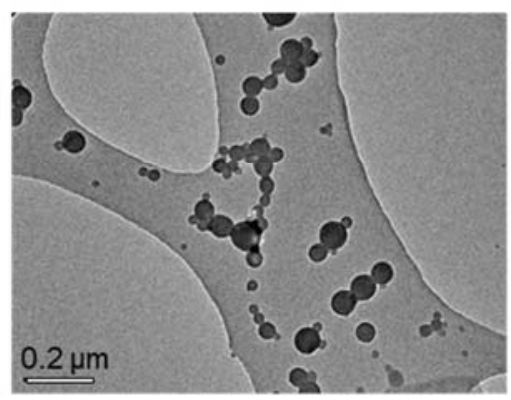

B

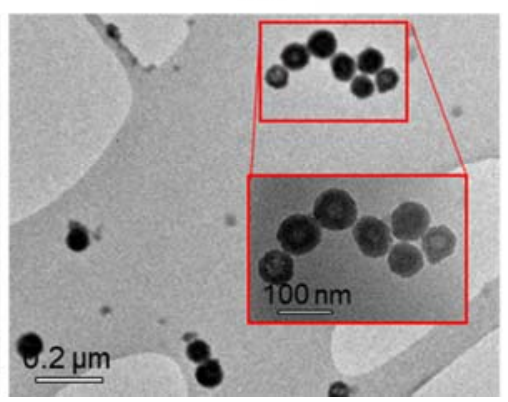

C

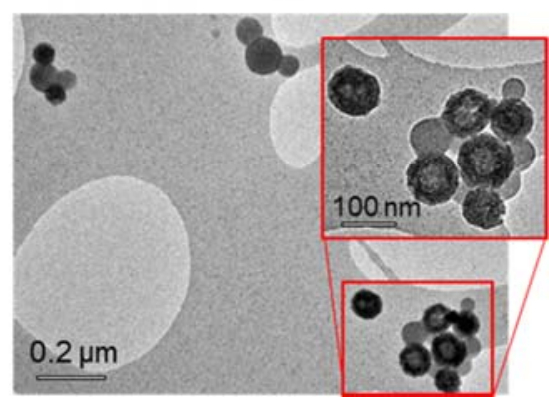

Figure 8. TEM images of FON dispersions deposited on holey carbon-coated copper grids. A) $\mathrm{FON}_{0,5}$ before irradiation, B) $\mathrm{FON}_{0,5}$ after irradiation, C) $\mathrm{FON}_{0}$ before irradiation at $365 \mathrm{~nm}$.

Interestingly, for sample $\mathrm{FON}_{0,5}$, all nanoparticles systematically appeared darker after photoirradiation, featuring a higher electronic contrast. This evolution could evoke denser systems, as a result of significant shrinkage due to photocrosslinking. When compared to model FON 0 , such geometry evolution is much less marked in agreement with little reactive species in the absence of TPO, indicating that dye degradation cannot be the sole pathway toward the noted structural change.

We thus performed atomic force microscopy measurements (AFM) to address at the nanometer scale some possible mechanical differences between non-photoirradiated and photoirradiated photocrosslinkable FONs. As FONs largely differ from rigid crosslinked polystyrene or silica nanoparticles often used as bioimaging probes, we decided to adopt an approach based on nanoparticle deformation upon compression rather than the common indentation method, especially valid on hard and non-deformable nanosystems. ${ }^{[38]}$ We thus used calibrated spherical glassy carbon AFM probes with tip radius $(100 \pm 10 \mathrm{~nm})$ comparable or higher than the radius of probed FONs, which favors compression instead of indentation during the conventional force curve measurements. In order to obtain reliable measurements, we carried 
out statistical analyses on a dozen of air-dried nanoparticles after deposition from highly diluted dispersions on cleaned mica surfaces, pretreated with lysine to favor FON attachment and avoid nanoparticle rolling effects. Under these controlled conditions, AFM force investigations of FON 0.5 containing the higher PT-SH molar fraction before and after photoirradiation displayed significant mechanical differences in terms of $E$ elastic modulus. $E$ values increased by $62 \%$ from non-photoirradiated FONs to photoirradiated ones, namely from $105 \pm 11$ to $170 \pm 10 \mathrm{MPa}$ (Figure 9).

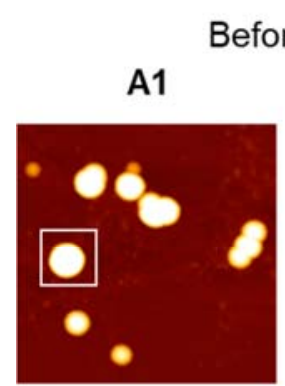

$1.5 \times 1.5 \mu \mathrm{m}$
B1

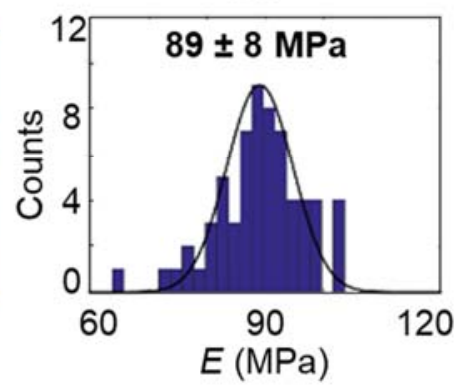

After irradiation

A2

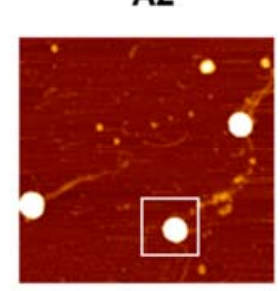

$1.5 \times 1.5 \mu \mathrm{m}$
B2

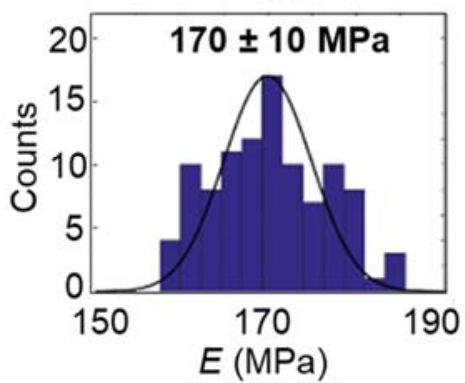

Figure 9. FON 0.5 (composition TPO:0.5 PT-SH:ALD) deposited on lysine-treated mica surface before (1) and after irradiation (2) at $365 \mathrm{~nm}$. A) AFM imaging of several nanoparticles. B) Elastic modulus $E$ distribution upon force mapping investigations of one single nanoparticle (selected by a white square on Figure 9A).

Such significant changes well match the expected mechanical evolution when going from softer nanostructures to stiffer ones, as expected upon photocrosslinking. The revisited exploration method with compression rather than indentation presented here for quantitative mechanical measurements of quite soft nanoparticles by means of AFM, appeared as a nice approach to inform on FON rigidification, as a consequence of photocrosslinking. 


\section{CONCLUSION}

These studies have evidenced the versatile approach of generating FONs devoid of surfactant and displaying colloidal stability by means of a novel model of micromixer, Microsphere500, allowing for straightforward variation of the mean diameter as a function of the flow velocities or stock solution concentration. Compared to classical manual addition, this flash nanoprecipitation technique yields highly concentrated FON dispersions, characterized with much smaller size, narrower polydispersity, and high fabrication reproducibility without causing subsequent precipitation. The fabrication of monocomposite FONs has successfully been extended to the microfluidic production of bicomposite FONs without causing demixing or precipitation into separated nanoparticle despite the high concentration used. Such coprecipitation process was proved by implying energy donors and acceptors amenable to efficient electronic energy transfer that led to considerably enhanced emission of red emitters as a result of the tight confinement and mutual polarity effects. Finally, we harnessed such confinement effects for the first time to our knowledge to promote reaction within FONs containing fluorophores with photopolymerizable units, and therefore obtain photocrosslinked FONs based on photoinitiated thiol-ene reaction. The results point out the difficult balance between photoinitiation, and fluorophore photodegradation upon irradiation due to competitive excitation absorption. Quantitative mechanical studies performed at the nanometer scale by resorting to AFM revealed FON stiffening after photoirradiation, confirming the possibility to perform reaction with self-assembled molecules. Future studies will explore the possibility of trapping or post-grafting various molecules of interest using coupled microfluidic devices to impart FONs with theranostic properties and high colloidal stability in biological media if required. Further investigations of the mixing dynamics within the Microsphere500 chamber, 
beyond the scope of the current studies, will also be performed by replacing the aluminum plates with optically transparent windows and using a camera with ultra-high acquisition rate.

\section{SUPPORTING INFORMATION}

Supporting Information is available from the Wiley Online Library or from the author.

\section{ACKNOWLEDGEMENTS}

Camille Alvarado and Angelina d'Orlando from the BIBS platform of Nantes-Angers INRA (France) are gratefully acknowledged for their kind advices regarding TEM image acquisition. The Cordouan Technologies Company (Bordeaux, France) is strongly acknowledged for its constant and rich support regarding the analyses of nanoparticle samples. CNRS and Nantes University are finally gratefully acknowledged for their essential financial support through the Interdisciplinary Challenge in Mechanobiology (ENAMEL project) and interdisciplinary call within the NExT i-site excellence initiative (METCIN project) respectively. Finally, PicoQuant $\mathrm{GmbH}$ (Berlin, Germany) is warmly thanked for its exceptional remote scientific and technological support.

KEYWORDS. Fluorescent organic nanoparticles • microfluidics $\bullet$ size and control modulation • photophysics $\bullet$ photochemistry

\section{CONFLICTS OF INTEREST}

There are no conflicts to declare.

\section{REFERENCES}

[1] a) S. J. Williams, C. Park, S. T. Wereley, Microfluid. Nanofluid. 2010, 8, 709-726; b) H. Ranchon, V. Picot, A. Bancaud, Sci. Rep. 2015, 5, 10128. 
[2] a) H. S. Peng, S. H. Huang, O. S. Wolfbeis, J. Nano. Res. 2010, 12, 2729-2733; b) H. Y. Zhou, M. Sharma, O. Berezin, D. Zuckerman, M. Y. Berezin, ChemPhysChem 2016, 17, 27-36.

[3] D. Wencel, T. Abel, C. McDonagh, Anal. Chem. 2014, 86, 15-29.

[4] a) M. Kanamala, W. R. Wilson, M. M. Yang, B. D. Palmer, Z. M. Wu, Biomaterials 2016, 85, 152-167; b) R. J. Gui, H. Jin, X. N. Bu, Y. X. Fu, Z. H. Wang, Q. Y. Liu, Coord. Chem. Rev. 2019, 383, 82-103.

[5] A. C. Anselmo, M. W. Zhang, S. Kumar, D. R. Vogus, S. Menegatti, M. E. Helgeson, S. Mitragotri, ACS Nano 2015, 9, 3169-3177.

[6] S. Patel, J. Kim, M. Herrera, A. Mukherjee, A. V. Kabanov, G. Sahay, Adv. Drug Delivery Rev. 2019, 144, 90-111.

[7] a) P. P. Mathai, J. A. Liddle, S. M. Stavis, Appl. Phys. Rev. 2016, 3, 011105; b) J. D. Keighron, A. G. Ewing, A. S. Cans, Analyst 2012, 137, 1755-1763; c) G. Chen, I. Roy, C. Yang, P. N. Prasad, Chem. Rev. 2016, 116, 2826-2885.

[8] a) R. Jenjob, F. Seidi, D. Crespy, Adv. Coll. Int. Sci. 2018, 260, 24-31; b) E. Lobry, F. Jasinski, M. Penconi, A. Chemtob, C. Croutxe-Barghorn, E. Oliveros, A. M. Braun, A. Criqui, RSC Adv. 2014, 4, 43756-43759.

[9] K. Landfester, Angew. Chem. Int. Ed. 2009, 48, 4488-4507.

[10] a) M. P. Robin, R. K. O'Reilly, Polymer Int. 2015, 64, 174-182; b) V. J. Pansare, M. J. Bruzek, D. H. Adamson, J. Anthony, R. K. Prud'homme, Mol. Imaging Biol. 2014, 16, 180 188.

[11] S. R. Mane, I. L. Hsiao, M. Takamiya, D. Le, U. Straehle, C. Barner-Kowollik, C. Weiss, G. Delaittre, Small 2018, 14, 1801571. 
[12] a) J. Mei, N. L. C. Leung, R. T. K. Kwok, J. W. Y. Lam, B. Z. Tang, Chem. Rev. 2015, 115, 11718-11940; b) S. Suzuki, S. Sasaki, A. S. Sairi, R. Iwai, B. Z. Tang, G.-i. Konishi, Angew. Chem. Int. Ed. 2020, 59, 9856-9867.

[13] a) J. Gierschner, L. Lüer, B. Milián-Medina, D. Oelkrug, H.-J. Egelhaaf, J. Phys. Chem. Lett. 2013, 4, 2686-2697; b) N. J. Hestand, F. C. Spano, Chem. Rev. 2018, 118, 7069-7163.

[14] a) E. Ishow, A. Brosseau, G. Clavier, K. Nakatani, P. Tauc, C. Fiorini-Debuisschert, S. Neveu, O. Sandre, A. Leaustic, Chem. Mater. 2008, 20, 6597-6599; b) J. Schill, A. Schenning, L. Brunsveld, Macromol. Rapid Commun. 2015, 36, 1306-1321; c) E. Genin, Z. H. Gao, J. A. Varela, J. Daniel, T. Bsaibess, I. Gosse, L. Groc, L. Cognet, M. BlanchardDesce, Adv. Mater. 2014, 26, 2258-2261; d) J. Ochi, K. Tanaka, Y. Chujo, Angew. Chem. Int. Ed. 2020, 59, 9841-9855.

[15] a) V. Privman, D. V. Goia, J. Park, E. Matijević, J. Coll. Int. Sci. 1999, 213, 36-45; b) W. S. Saad, R. K. Prud'homme, Nano Today 2016, 11, 212-227.

[16] B. K. Johnson, R. K. Prud'homme, AIChE J. 2003, 49, 2264-2282.

[17] a) N. M. Pinkerton, M. E. Gindy, V. L. Calero-DdelC, T. Wolfson, R. F. Pagels, D. Adler, D. Gao, S. Li, R. Wang, M. Zevon, N. Yao, C. Pacheco, M. J. Therien, C. Rinaldi, P. J. Sinko, R. K. Prud'homme, Adv. Healthcare Mater. 2015, 4, 1376-1385; b) Y. Liu, C. Y. Cheng, Y. Liu, R. K. Prud'homme, R. O. Fox, Chem. Eng. Sci. 2008, 63, 2829-2842.

[18] M. Akbulut, P. Ginart, M. E. Gindy, C. Theriault, K. H. Chin, W. Soboyejo, R. K. Prud'homme, Adv. Funct. Mater. 2009, 19, 718-725.

[19] a) A. Belkadi, A. Montillet, J. Bellettre, J. Energy Res. Tech. 2018, 140, 012202; b) E. A. Melo-Espinosa, J. Bellettre, D. Tarlet, A. Montillet, R. Piloto-Rodriguez, S. Verhelst, Fuel 2018, 219, 320-330. 
[20] A. Belkadi, D. Tarlet, A. Montillet, J. Bellettre, P. Massoli, Int. J. Multiphase Flow 2015, $72,11-23$.

[21] a) J. Boucard, T. Briolay, T. Blondy, M. Boujtita, S. Nedellec, P. Hulin, M. Grégoire, C. Blanquart, E. Ishow, ACS Appl. Mater. Int. 2019, 11, 32808-32814; b) J. Su, T. Fukaminato, J.-P. Placial, T. Onodera, R. Suzuki, H. Oikawa, A. Brosseau, F. Brisset, R. Pansu, K. Nakatani, R. Métivier, Angew. Chem. Int. Ed. 2016, 55, 3662-3666; c) E. Campioli, C. Rouxel, M. Campanini, L. Nasi, M. Blanchard-Desce, F. Terenziani, Small 2013, 9, 19821988.

[22] A. Kaeser, I. Fischer, R. Abbel, P. Besenius, D. Dasgupta, M. A. J. Gillisen, G. Portale, A. L. Stevens, L. M. Herz, A. Schenning, ACS Nano 2013, 7, 408-416.

[23] S. Olivier, L. Derue, B. Geffroy, T. Maindron, E. Ishow, J. Mater. Chem. C 2015, 3, 84038412.

[24] L. Derue, S. Olivier, D. Tondelier, T. Maindron, B. Geffroy, E. Ishow, ACS Appl. Mater. \& Interfaces 2016, 8, 16207-16217.

[25] P. J. Skrdla, J. Phys. Chem. A 2009, 113, 9329-9336.

[26] V. Genot, S. Desportes, C. Croushore, J. P. Lefevre, R. B. Pansu, J. A. Delaire, P. R. von Rohr, Chem. Eng. J. 2010, 161, 234-239.

[27] Y.-C. Tan, V. Cristini, A. P. Lee, Sens. Actuators B-Chem 2006, 114, 350-356.

[28] K. Roger, B. Cabane, Angew. Chem. Int. Ed. 2012, 51, 5625-5628.

[29] J. Boucard, C. Linot, T. Blondy, S. Nedellec, P. Hulin, C. Blanquart, L. Lartigue, E. Ishow, Small 2018, 14, 1802307.

[30] M. Breton, G. Prevel, J. F. Audibert, R. Pansu, P. Tauc, B. Le Pioufle, O. Francais, J. Fresnais, J. F. Berret, E. Ishow, Phys. Chem. Chem. Phys. 2011, 13, 13268-13276. 
[31] E. Ishow, R. Guillot, G. Buntinx, O. Poizat, J. Photochem. Photobiol. A: Chem. 2012, 234, 27-36.

[32] J. Schill, L. Ferrazzano, A. Tolomelli, A. Schenning, L. Brunsveld, RSC Adv. 2020, 10, 444-450.

[33] a) U. Gosele, M. Hauser, U. K. A. Klein, R. Frey, Chem. Phys. Lett. 1975, 34, 519-522; b) K. K. Pandey, H. C. Joshi, T. C. Pant, Chem. Phys. Lett. 1988, 148, 472-478.

[34] a) N. Corrigan, J. Yeow, P. Judzewitsch, J. Xu, C. Boyer, Angew. Chem. Int. Ed. 2019, 58, 5170-5189; b) E. Blasco, M. Wegener, C. Barner-Kowollik, Adv. Mater. 2017, 29, 1604005.

[35] T. E. Brown, K. S. Anseth, Chem. Soc. Rev. 2017, 46, 6532-6552.

[36] a) O. Z. Durham, D. V. Chapman, S. Krishnan, D. A. Shipp, Macromolecules 2017, 50, 775-783; b) D. W. Yee, M. D. Schulz, R. H. Grubbs, J. R. Greer, Adv. Mater. 2017, 29, 1605293.

[37] S. Dadashi-Silab, S. Doran, Y. Yagci, Chem. Rev. 2016, 116, 10212-10275.

[38] M. E. Dokukin, I. Sokolov, Macromolecules 2012, 45, 4277-4288. 


\section{TABLE OF CONTENTS GRAPHIC}

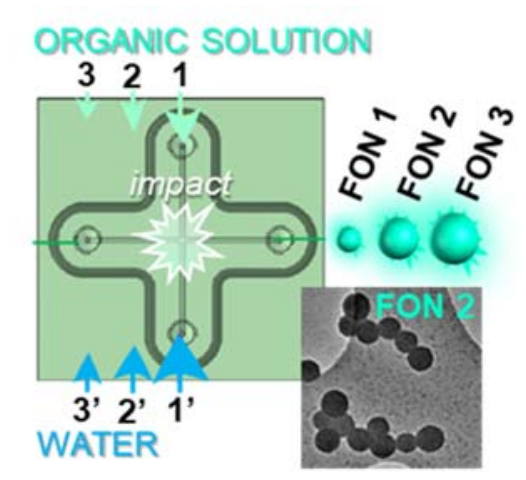

Keywords: Fluorescent organic nanoparticles $\bullet$ microfluidics $\bullet$ size and control modulation • photophysics $\bullet$ photochemistry

Authors: Stéphane Hoang, Simon Olivier, Stéphane Cuenot, Agnès Montillet, Jérôme Bellettre, and Eléna Ishow*

Title: Microfluidic Assisted Flash Precipitation of Photocrosslinkable Fluorescent Organic Nanoparticles for Fine Size Tuning and Enhanced Photoinduced Processes

Dispersions of bright fluorescent organic nanoparticles are fabricated at high concentration using high-impact mixing chamber allowing for fine size tuning and narrow size distribution. Dye spatial confinement within bicomposite nanoparticles enables amplified emission upon energy transfer due to polarity effects, and photopolymerization addressed at the nanoscale by atomic force microscopy. 